\title{
Design of Micro-strip Symmetrical Dual- band Filter Based on Wireless Sensor Network Nodes
}

\author{
Wenbo Cheng ${ }^{1,2^{*}}$ (D) Kai Deng ${ }^{1}$ and Wei Cheng ${ }^{3}$
}

\begin{abstract}
The micro-strip antenna filter design of wireless sensor network nodes is usually used to improve the out-of-band suppression and frequency selectivity by increasing the order of the filters, but the filters are usually single band, not only the size is large, but also the in-band characteristics of the filters are not ideal. This paper proposes a method to design a micro-strip symmetric dual-band filter in wireless sensor network nodes. Firstly, the coupling matrix of singlepassband filter is obtained by using the synthesis method of generalized Chebyshev filter function. Then, the coupling matrix of the dual-passband filter is generated according to the reflection zeros and the transmission zeros. Finally, the $S$ parameters response curve is drawn by mapping the normalized frequency domain of the dual-passband to the actual frequency domain. According to the data analysis and experimental results, the method is feasible and effective to design a micro-strip symmetrical dual-band filter. It can not only provide a more guiding design method for the joint design of antenna and RF front-end circuit, but also realize the spread of single-passband filter to multi-frequency for a wireless sensor network node antenna.
\end{abstract}

Keywords: Wireless sensor network nodes, Symmetrical dual-passband technology, Micro-strip antenna, Generalized Chebyshev function, Cross-coupling synthesis theory, Transmission zeros

\section{Introduction}

The traditional radio frequency $(\mathrm{RF})$ transceiver system consists of antenna, filter, power amplifier, low noise amplifier, and other devices, which often work under a single communication standard. If multiple communication standards run at the same time, it requires multiple independenttransceiver systems to form a parallel working system which will be larger in size, high in power consumption and high in cost, and has been unable to meet the application needs of wireless sensor network nodes in the era of the big data, which has attracted the attention and widespread concern of researchers all over the world [1-13].

Micro-strip filter is a very important component of wireless sensor network (WSN) nodes, which is used to select useful signals and suppress clutter interference

\footnotetext{
*Correspondence: tgzy09@126.com

'School of Physics and Electronic Engineering, Yibin University, Ybin 644000, China

${ }^{2}$ Key Lab of Earth Exploration \& Information Techniques of Ministry of Education, Chengdu University of Technology, Chengdu 610059, China Full list of author information is available at the end of the article
}

signals. Multi-frequency micro-strip filters are required to effectively pick up the signals of each separate frequency band and prevent signal crosstalk between adjacent channels, which requires higher frequency selectivity and out-of-band rejection of the filters [4-6]. In recent years, scholars in various countries have carried out in-depth research and proposed various design methods to solve the two core problems in the design of multi-band micro-strip filters [14-20]: Firstly, multi-point frequency selection can be realized, multi-frequency can work in parallel, and each central frequency point has a certain capacity bandwidth; secondly, the signals in adjacent frequency bands must be effectively isolated and can not interfere with each other, and the out-of-band rejection performance of the filter is reliable. There are two main methods for designing multi-band filters [21-35]: Firstly, based on the combination of multi-band filters, including the cascade of broadband filters and notch filters, and the parallel connection of multiple filters in different frequency bands; secondly, based 
on the parasitic frequency of resonators, a multi-band filter is designed.

Traditional Butterworth, Chebyshev, and elliptic function filters can increase the design order of the filter to improve the filter's out-of-band suppression and frequency selectivity, but the designed filter is usually single-band, not only the size is large, but also the filter's in-band characteristics are not ideal [36]. Generalized Chebyshev filter (GCF) is also known as quasi-elliptic function filter. It is between Chebyshev and elliptic function filter, which has excellent in-band characteristics and steep edge characteristics $[37,38]$. The transmission zeros of generalized Chebyshev filter can be flexibly controlled, which can be used to improve filter selectivity and stop-band isolation. In order to improve the in-band and out-of-band performance of the filter, the communication band of the filter is extended from a single band to a dual band. Based on the synthesis theory of generalized Chebyshev filter, the frequency transformation is carried out with low-frequency prototype, and the design from low-frequency prototype to dual-band filter is realized with a cross-coupling synthesis theory.

\section{Methodology}

\subsection{Frequency conversion method of symmetrical dual} band

The single-band filter model is transformed into a dual-band filter and has to undergo two frequency conversion. Firstly, the normalized single low-pass filter is transformed into a normalized dual-band filter, and then, the normalized dual-band filter is transformed into a dual-band filter of actual frequency by one transformation. Figure 1 shows that three frequency variables are applied in the frequency conversion process, one is the normalized low-pass frequency variable $\Omega$, the other is the dual-band normalized intermediate variable $\Omega^{\prime}$, and the third is the actual frequency variable $\omega$.

The transmission function of the generalized Chebyshev $\mathrm{N}$-order low-pass filter [35] is shown as below

$$
T^{2}(s)=S_{21}^{2}(s)=\frac{1}{1+\varepsilon^{2} C_{N}^{2}(s)}
$$

Among them, $s=j \Omega, \varepsilon$ is the ripple coefficients in the passband and $C_{N}(s)$ is the characteristic function of the generalized Chebyshev low-pass filter.

The transmission zeros and reflection zeros of the generalized Chebyshev low-pass filter are described.

$$
C_{N}(\omega)=\frac{F(\omega)}{P(\omega)}=\frac{\prod_{i=1}^{N}\left(s-s_{p i}\right)}{\prod_{j=1}^{K}\left(s-s_{z j}\right)}(K \leq N)
$$

Among them, $s_{p i}$ is the transmission pole, also known as the reflection zero, that is the filter power optimal transmission point and $s_{z j}$ is the transmission zero.

It is assumed that the transmission function of the generalized Chebyshev low-pass filter is about $\Omega$ axis symmetry. The dual-band frequency conversion process is shown in Fig. 1. Thus, the number of transmission zeros and reflection zeros of the generalized Chebyshev dual-band filter obtained by frequency transformation is $2 K$ and $2 N$, respectively. The low passband frequency range $j s^{\prime}$ is $-j$ to $-j \Omega_{k}^{\prime}$, and the high passband frequency range $j s^{\prime}$ is $j \Omega_{k}^{\prime}$ to $j$.

Firstly, the normalized frequency conversion from single passband to double passband is realized. The frequency conversion equations such as Eqs. (3) and (4) are given.

$$
\begin{array}{ll}
s=\frac{s^{\prime}}{a_{1}}+\frac{a_{2}}{s^{\prime}} & \left(\Omega^{\prime}>0\right) \\
s=-\left(\frac{s^{\prime}}{a_{1}}+\frac{a_{2}}{s^{\prime}}\right) & \left(\Omega^{\prime}<0\right)
\end{array}
$$

$s^{\prime}$ is a frequency variable that is mapped from the prototype $s$ plane. As shown in Fig. 1, when $s$ changes from normalized frequency -1 to 1 in the $\Omega$ domain, it maps to $s$ from normalized frequency $\Omega_{k}^{\prime}$ to 1 in the $\Omega^{\prime}>0$ domain; when $s$ changes from normalized

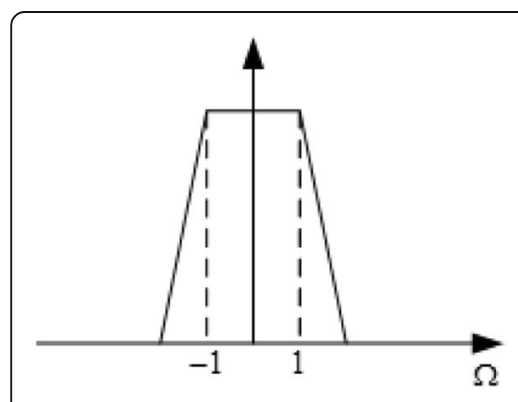

(a)

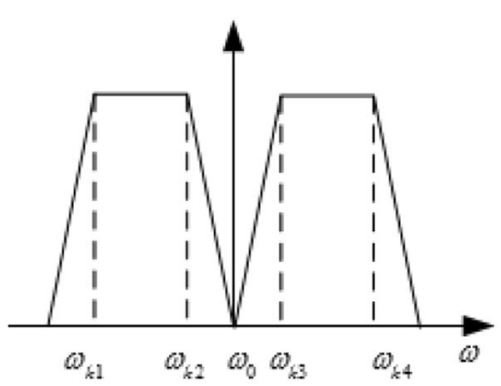

(b)

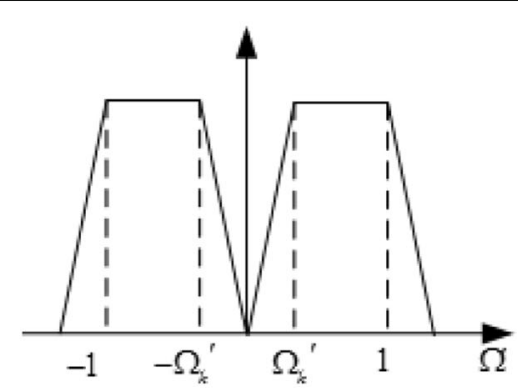

(c)

Fig. 1 Schematic diagram of dual-passband frequency conversionb. a The $\Omega$ domain. b The $\Omega$ ' domain. c The $\omega$ domain 
frequency -1 to 1 in the $\Omega$ domain, it maps to $s$ from normalized frequency $\Omega_{k}^{\prime}$ to 1 in the $\Omega^{\prime}>0$ domain. Degenerate (3) into

$$
s^{\prime 2}-s a_{1} s^{\prime}+a_{1} a_{2}=0
$$

The following Eq. (6) can be obtained by expression (5) of which $s=j \Omega$.

$$
s^{\prime}=\frac{j \Omega a_{1} \pm j a_{1} \sqrt{(\Omega)^{2}+\frac{4 a_{2}}{a_{1}}}}{2}
$$

When $s=j, s^{\prime}=j$ or when $s=-j, s^{\prime}=j \Omega_{k}^{\prime}$, the Eq. (7) can be obtained from the Eq. (6).

$$
1=\frac{a_{1}}{2}\left(1+\sqrt{1+4 a_{2} / a_{1}}\right) \Omega_{k}{ }^{\prime}=\frac{a_{1}}{2}\left(1-\sqrt{1+4 a_{2} / a_{1}}\right)
$$

We can get $s=j$ when $s^{\prime}=j$ and $s^{\prime}=-j \Omega_{k}^{\prime}, s=-j$ when $s^{\prime}=-j$ and $s^{\prime}=j \Omega_{k}^{\prime}$ from Fig. 1. Therefore, the following expressions can be obtained.

$$
a_{1}=1-\Omega_{k}{ }^{\prime} \quad, \quad a_{2}=\frac{\Omega_{k}{ }^{\prime}}{1-\Omega_{k}{ }^{\prime}}
$$

Thus, the normalized single passband to normalized dual-band frequency conversion can be easily realized by expressions (7) and (8).

Secondly, the normalized dual-band to the actual frequency of the dual-band transformation, that is, the frequency transform domain from $\Omega^{\prime}$ domain to $\omega$ domain transformation. The transformation equation is

$$
s^{\prime}=\frac{\varpi}{l_{1}}+\frac{l_{2}}{\varpi}(\omega>0)
$$

Among it, $\varpi=j \omega$. We can see that values of $1, \Omega_{k}^{\prime}$, $\Omega_{k}^{\prime}$, and -1 in the $\Omega^{\prime}$ domain are mapped, respectively, to $\omega_{k 4}, \omega_{k 3}, \omega_{k 2}$, and $\omega_{k 1}$ of the $\omega$ domain from Fig. 1 .

We can get the expressions of $l_{1}, l_{2}$, and $\Omega_{k}^{\prime}$.

$$
l_{1}=\omega_{k 4}-\omega_{k 1}, \quad l_{2}=\frac{\omega_{k 4} \omega_{k 1}}{\omega_{k 4}-\omega_{k 1}}, \quad \Omega_{k}{ }^{\prime}=\frac{\omega_{k 3}-\omega_{k 2}}{\omega_{k 4}-\omega_{k 1}}
$$

\subsection{Synthesis method of $\mathrm{N}$-order cross-coupling matrix}

With the development of communication technology, the spectrum is becoming more and more crowded, and the technical specifications of the filter, especially the rectangular requirements, are becoming more and more stringent. Traditional Butterworth and Chebyshev filters have been unable to meet the requirements. In order to improve the selectivity and out-of-band isolation of filters, transmission zeros are usually introduced into filters, which are generated by cross-coupling between non-adjacent resonators [30-35]. The cross-coupling filter with finite transmission zeros is the most common choice. Generalized Chebyshev function is usually used to implement it.

The lumped parameter equivalent circuit and equivalent network parameters of the coupling filter are shown in Fig. 2a and b, respectively. According to the Kirchhoff theorem that the sum of the voltage is

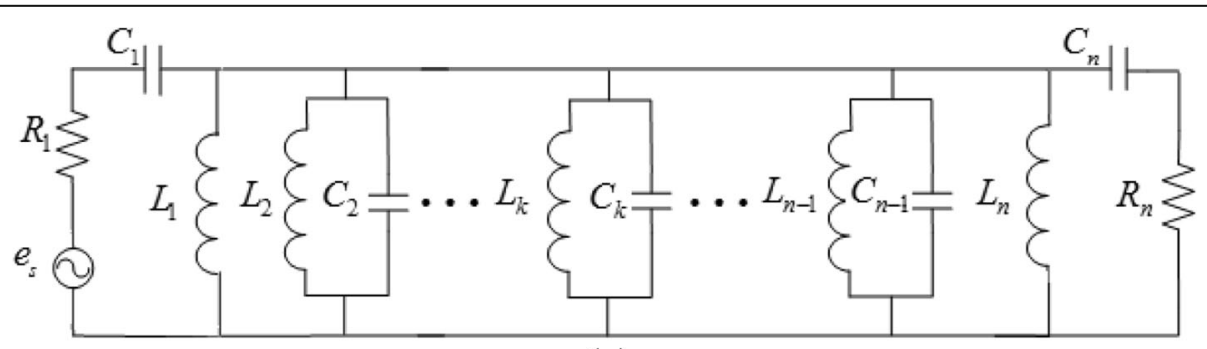

(a)

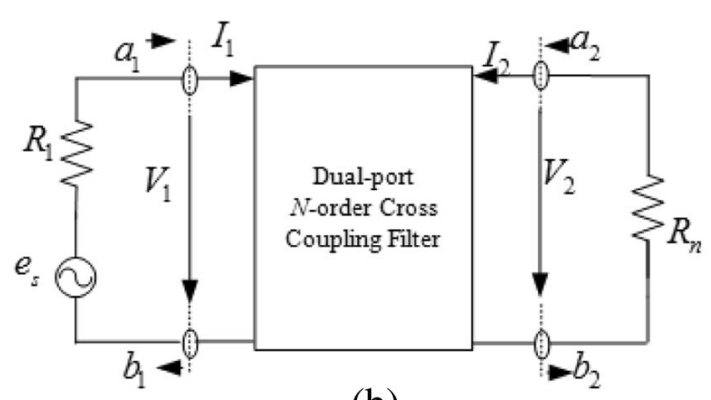

(b)

Fig. 2 Equivalent circuit (a) and equivalent network parameters (b) of the loop equation of lumped parameter of $\mathrm{N}$-order coupling filter 
Table 1 Polynomial roots of the four-order single-passband filter

\begin{tabular}{llll}
\hline Functions & $\begin{array}{l}\text { Transmission zeros, } \\
\text { the roots of } P(\Omega)\end{array}$ & $\begin{array}{l}\text { Reflection zeros, } \\
\text { the roots of } F(\Omega)\end{array}$ & $\begin{array}{l}\text { Transmitting or reflecting } \\
\text { singularities, the roots of } E(\Omega)\end{array}$ \\
\hline 1 & $-1.5 j$ & $-0.9424 j$ & $-0.7964+1.020 j$ \\
2 & $1.5 j$ & $0.9424 j$ & $-1.2310+0.1914 j$ \\
3 & $-\infty$ & $-0.4299 j$ & $0.7964+1.020 j$ \\
4 & $\infty$ & $0.4299 j$ & $1.2310+0.1914 j$ \\
\hline
\end{tabular}

zero along the loop, the voltage of each loop is described Eq. (11).

$$
\left\{\begin{array}{l}
\left(R_{1}+j \omega L_{1}+1 / j \omega C_{1}\right) \cdot i_{1}-j \mathrm{~L}_{12} \cdot i_{2} \cdots-j L_{1 n} \cdot i_{n}=e_{s} \\
-j L_{12} \cdot i_{1}+\left(j \omega L_{2}+1 / j \omega C_{2}\right) \cdot i_{2}-j L_{2 n} \cdot i_{n}=0 \\
\vdots \\
-j L_{n 1} \cdot i_{1}-j L_{n 2} \cdot i_{2} \cdots+\left(R_{2}+j \omega L_{n}+1 / j \omega C_{n}\right) \cdot i_{n}=0
\end{array}\right.
$$

The specific method of treatment is shown in document [15], Eq. (11) which is expressed by matrix as Eq. (12).

$$
[Z] \cdot[I]=[e]
$$

[Z] is $N \times N$ impedance matrix. Each resonator of synchronous tuning filter has the same resonance frequency $\omega_{0}=1 / \sqrt{L C}$, and among it, $L=L_{1}=L_{2}=\cdots L_{n}, C=C_{1}=$ $C_{2}=\cdots C_{n} . F B W=\Delta \omega / \omega_{0}$ is the relative bandwidth. The normalized impedance matrix is $[\bar{Z}]=[Z] /\left(\omega_{0} L \cdot F B W\right)$.

$$
\begin{aligned}
{[\bar{Z}]=} & {\left[\begin{array}{cccc}
\frac{R_{1}}{\omega_{0} L \cdot F B W}+p & -j \frac{\omega L_{12}}{\omega_{0} L \cdot F B W} & \cdots & -j \frac{\omega L_{1 n}}{\omega_{0} L \cdot F B W} \\
-j \frac{\omega L_{21}}{\omega_{0} L \cdot F B W} & p & \cdots & \vdots \\
-j \frac{\omega L_{n 1}}{\omega_{0} L \cdot F B W} & -j \frac{\vdots}{\omega_{0} L \cdot F B W} & \cdots & \frac{R_{n}}{\omega_{0} L \cdot F B W}+p
\end{array}\right] } \\
& p=j \frac{1}{F B W}\left(\frac{\omega}{\omega_{0}}-\frac{\omega_{0}}{\omega}\right)
\end{aligned}
$$

The external quality factor is defined as $Q_{e i}=R_{i} / \omega_{0} L(i$ $=1, N)$ and the coupling coefficient is $M_{i j}=L_{i j} / L$. While $\omega_{0} / \omega \approx 1$ in the narrowband filter, there are

$$
[\bar{Z}]=\left[\begin{array}{cccc}
\frac{1}{q_{e i}}+p & -j m_{12} & \cdots & -j m_{1 n} \\
-j m_{21} & p & \cdots & \vdots \\
\vdots & \vdots & \vdots & \vdots \\
-j m_{n 1} & -j m_{n 2} & \cdots & \frac{1}{q_{e n}}+p
\end{array}\right]
$$

Among it, $q_{e i}=Q_{e i} \cdot F B W(i=1.2), \quad m_{i j}=M_{i j} / F B W$, to $I_{1}=i_{1}, \quad I_{n}=-i_{n}$ in Fig. 2b, then we can get the Eq. (15).

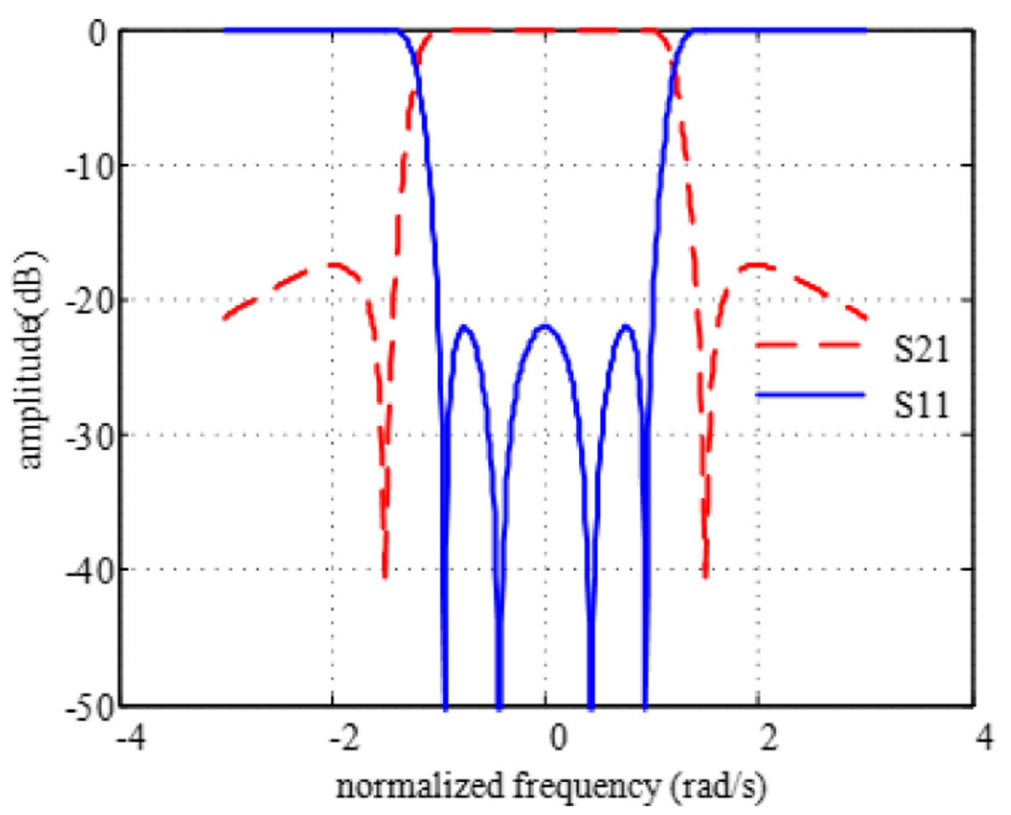

Fig. 3 s parameters curve of four-order single-passband filter 


$$
\begin{array}{ll}
a_{1}=\frac{e_{s}}{2 \sqrt{R_{1}}} & b_{1}=\frac{e_{s}-2 i_{1} R_{1}}{2 \sqrt{R_{1}}} \\
a_{2}=0 & b_{2}=i_{n} \sqrt{R_{n}}
\end{array}
$$

After synthesizing the coupling matrix, the coupling matrix is deformed to correspond to the actual circuit model structure. This is advantageous to the actual physical circuit design, in which the negative coupling coefficient in the coupling matrix represents the capacitance coupling in the cross-coupling of the adjacent resonator circuit, and the positive coupling coefficient in the coupling matrix represents the inductive coupling in the cross-coupling of the adjacent resonator circuit.

\section{Results and discussion}

\subsection{Basic parameters of dual-band filter}

Based on the previous generalized Chebyshev filter function synthesis theory of dual-band filter, a symmetrical dual-band filter (SDF) are designed. Assuming that the frequency range of the low passband is $2.9-2.95 \mathrm{GHz}$, the frequency range of the high passband is $3.05-3.10$ $\mathrm{GHz}$ and the in-band return loss is $\mathrm{RL}=22 \mathrm{~dB}$.

The dual-band is symmetrical with respect to the center of $\omega_{0}=3 \mathrm{GHz}$, and the four transmission zeros of the single-pass low-pass filter are $1.5 j,-1.5 j, j \infty$, and $-j \infty$.

$\omega_{k 1}=2.90, \quad \omega_{k 2}=2.95, \quad \omega_{k 3}=3.05$, and $\omega_{k 4}=3.10$ can be calculated from the conditions given above. According to Eq. (10), we can obtain $\Omega_{k}^{\prime}=0.5$. According to Eq. (8), we can obtain $a_{1}=0.5$ and $a_{2}=1$.
3.2 Design and simulation of symmetrical dual-band filter Based on the generalized Chebyshev filter synthesis method described in the previous section, we can obtain the roots of these functions $P(\Omega), \quad F(\Omega)$, and $E(\Omega)$, as shown in Table 1.

$$
\begin{aligned}
& P(\Omega)=\Omega^{2}-2.25 \\
& F(\Omega)=\Omega^{4}-1.730 \Omega^{2}+0.1641 \varepsilon-0.8666 \\
& E(\Omega)=\Omega^{4}-j 2.423 \Omega^{3}-4.009 \Omega^{2}+j 3.81 \Omega+2.601
\end{aligned}
$$

We can plot the response curve of the $S$ parameter, as shown in Fig. 3. The coupling matrix [16] deduced from short-circuit admittance parameters is:

$$
m=\left[\begin{array}{cccc}
0 & 0.861 & 0 & -0.476 \\
0.861 & 0 & -0.911 & 0 \\
0 & 0.911 & 0 & -0.861 \\
-0.476 & 0 & -0.861 & 0
\end{array}\right]
$$

Now, by using the frequency mapping equation, the reflection and transmission zeros of single-passband normalized generalized Chebyshev function filters and $a_{1}=0.5$, $a_{2}=1$ are substituted in Eq. (6), we can obtain:

$$
s^{\prime}=\frac{j \Omega a_{1} \pm j a_{1} \sqrt{(\Omega)^{2}+\frac{4 a_{2}}{a_{1}}}}{2}=\frac{j 0.5\left(\Omega \pm \sqrt{(\Omega)^{2}+8}\right)}{2}
$$

The transmission and reflection zeros of dual-band filters are obtained by using Eq. (16). Then, the root of

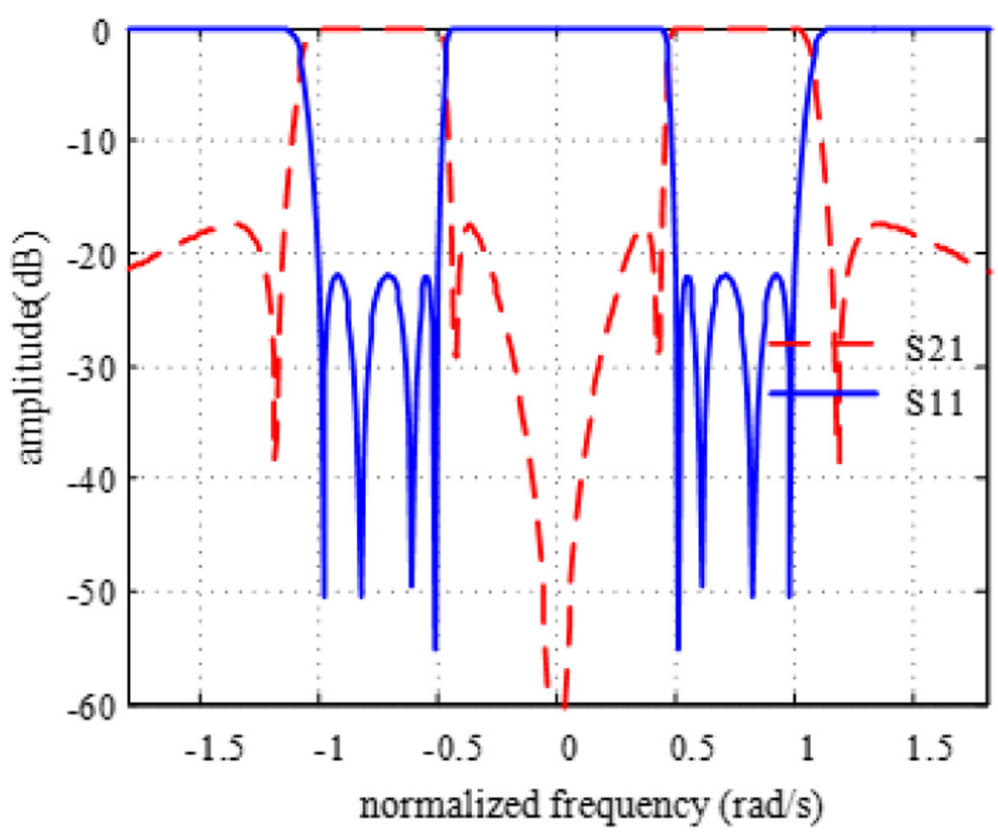

Fig. 4 Normalized S parameters response curve of dual-passband filter 


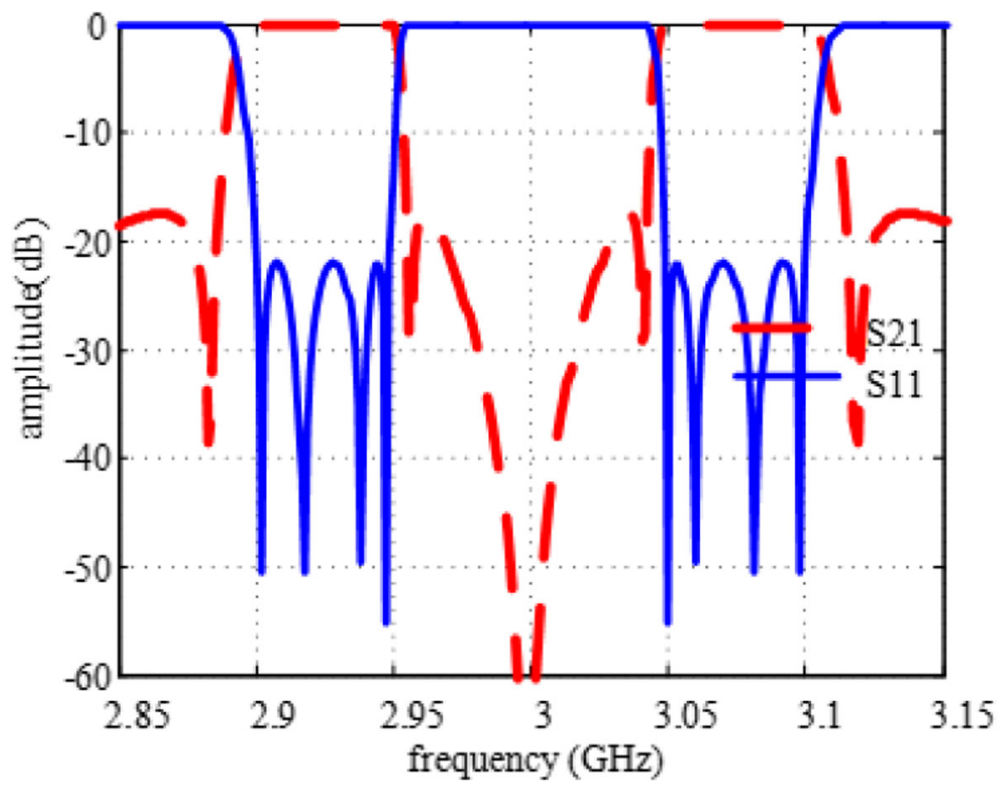

Fig. 5 S parameters response curve of dual-passband actual frequency

$E\left(s^{\prime}\right)$ is obtained by using the generalized Chebyshev filter function synthesis of a symmetrical dual-band. The roots of $P\left(\Omega^{\prime}\right), F\left(\Omega^{\prime}\right)$, and $E\left(\Omega^{\prime}\right)$ are listed in Table 2.

$$
\begin{aligned}
P\left(\Omega^{\prime}\right)= & \Omega^{\prime 6}+1.5625 \Omega^{\prime 4}+0.25 \Omega^{\prime 2} \\
F\left(\Omega^{\prime}\right)= & \Omega^{\prime 8}+2.2682 \Omega^{\prime 6}+1.7785 \Omega^{\prime 4}+0.56706 \Omega^{\prime 2} \\
& +0.0625 \\
E\left(\Omega^{\prime}\right)=\Omega^{\prime 8}+1.119 \Omega^{\prime 7}+2.895 \Omega^{\prime 6}+2.087 \Omega^{\prime 5}+2.524 \Omega^{\prime 4} & \\
& +1.044 \Omega^{\prime 3}+0.723 \Omega^{\prime 2}+0.1399 \Omega^{\prime 1}+0.062
\end{aligned}
$$

Especially, the filter is a four-order filter, with two finite transmission zeros, and two transmission zeros located at the positive and negative infinity. In the case of dual-passband transformation, the transmission zeros at infinity of single passband correspond to the transmission zeros at zero of dual passband. After the transmission and reflection zeros of the two passbands are obtained, the expressions of each polynomial of the two passbands can be obtained by using the synthesis method of the generalized Chebyshev filter function.

Thus, the normalized response curve of the dual band-pass filter can be obtained, as shown in Fig. 4.

Finally, Eq. (10) is used to calculate $l_{1}$ and $l_{2}$, and the Eq. (17) are used to map the frequency domain from $\Omega$ to $\omega$ domain. Then, the $S$ parameter curve in the actual frequency domain is obtained as shown in Fig. 5.

$$
\omega=\frac{l_{1}}{2}\left(\Omega^{\prime}+\sqrt{1+4 l_{2} / l_{1}}\right)
$$

\begin{tabular}{|c|c|c|c|}
\hline Functions & $\begin{array}{l}\text { Transmission zeros, } \\
\text { the roots of } P\left(\Omega^{\prime}\right)\end{array}$ & $\begin{array}{l}\text { Reflection zeros, } \\
\text { the roots of } F(\Omega)\end{array}$ & $\begin{array}{l}\text { Transmitting or reflecting singularities, } \\
\text { the roots of } E\left(\Omega^{\prime}\right)\end{array}$ \\
\hline 1 & $1.175 j$ & $0.5097 j$ & $-0.0656+1.066 j$ \\
\hline 2 & $0.4254 j$ & $-0.9809 j$ & $-0.0656-1.066 j$ \\
\hline 3 & $-0.4254 j$ & $0.9809 j$ & $-0.1711+0.5115 j$ \\
\hline 4 & $-1.175 j$ & $-0.5097 j$ & $-0.2941+0.8791 j$ \\
\hline 5 & 0 & $0.6078 j$ & $-0.2941-0.8791 j$ \\
\hline 6 & 0 & $-0.6078 j$ & $-0.0287-0.4673 j$ \\
\hline 7 & - & $-0.8227 j$ & $-0.1711-0.5115 j$ \\
\hline 8 & - & $0.8227 j$ & $-0.0287+0.4673 j$ \\
\hline
\end{tabular}

The coupling matrix obtained from the coefficients of $P\left(\Omega^{\prime}\right), \quad F\left(\Omega^{\prime}\right)$, and $E\left(\Omega^{\prime}\right)$ are then rotated to eliminate

Table 2 Polynomial roots of the eight-order dual-band filter 
the element. The folded $N+2$-order, that is, the tenth-order coupling matrix is.

$m=\left[\begin{array}{ccccccccccc}0 & 0.7481 & 0.0000 & 0.0000 & 0.0000 & 0.0000 & 0.0000 & 0.0000 & 0.0000 & 0 \\ 0.7481 & 0.0000 & 0.8215 & -0.0000 & 0.0000 & 0.0000 & -0.0000 & -0.0000 & -0.2045 & 0.0000 \\ 0.0000 & 0.8215 & -0.0000 & 0.4075 & 0 & 0 & 0 & 0.1129 & 0.0000 & 0.0000 \\ 0.0000 & 0.0000 & 0.4075 & 0 & 0.4750 & 0 & 0.5746 & 0.0000 & -0.0000 & 0.0000 \\ 0.0000 & 0.0000 & -0.0000 & 0.4750 & 0.0000 & -0.2519 & 0.0000 & -0.0000 & 0.0000 & 0.0000 \\ 0.0000 & -0.0000 & 0.0000 & 0.0000 & -0.2519 & 0.0000 & 0.4750 & 0.0000 & 0.0000 & -0.0000 \\ 0.0000 & 0.0000 & -0.0000 & 0.5746 & -0.0000 & 0.4750 & -0.0000 & 0.4075 & -0.0000 & 0.0000 \\ 0.0000 & 0.0000 & 0.1129 & 0.0000 & -0.0000 & 0.0000 & 0.4075 & -0.0000 & 0.8215 & -0.0000 \\ 0.0000 & -0.2045 & -0.0000 & -0.0000 & 0.0000 & 0.0000 & 0.0000 & 0.8215 & 0.0000 & 0.7481 \\ 0 & 0.0000 & 0.0000 & 0.0000 & 0.0000 & -0.0000 & 0.0000 & -0.0000 & 0.7481 & 0\end{array}\right]$

\section{Conclusion}

This paper presented an experimental study on the micro-strip dual-band filter based on wireless sensor network nodes. Firstly, according to the order, the position of the transmission zeros, and the ripple coefficients in the band of the filter to be designed, the generalized Chebyshev filter function synthesis method is applied to synthesize a single-passband filter by combining the relationship between the short-circuit admittance parameters. The coupling matrix of the singlepassband filter is obtained. Secondly, the generalized Chebyshev function polynomial is constructed according to the symmetrical frequency conversion equations from single-band to dual-band, and the coupling matrix of the dual-band filter is synthesized by the relationship between the generalized Chebyshev function polynomial and the short-circuit admittance parameters. Finally, the normalized frequency domain is mapped to the actual frequency domain, and the $S$ parameter response curve in the actual frequency domain is obtained. By flexibly controlling the transmission zeros of the generalized Chebyshev filter, the selectivity and stop-band isolation of the filter can be effectively improved, and this method can design multi-band filters with excellent performance and has a positive guiding role in the physical circuit design of micro-strip filters based on wireless sensor network nodes.

\section{Abbreviations}

GCF: Generalized Chebyshev filter; RF: Radio frequency; SDF: Symmetrical dual-band filter; WSN: Wireless sensor networks

\section{Acknowledgements}

The research presented in this paper was supported by National Natural Science Foundation, Sichuan Provincial Education Department and Yibin University, China.

\section{Funding}

The authors acknowledge the Scientific Research Fund of Sichuan Provincial Education Department (Grant: 14ZA0269), Scientific Research Key Project of
Yibin University (Grant: 2013QD02) and the National Natural Science Foundation of China (Grant: 61201266).

\section{Availability of data and materials}

The simulation code can be downloaded by contacting author after three years of publication. Mostly, I got the writing material from different journals as presented in the references. MATLAB tool has been used to simulate my concept.

\section{Authors' contributions}

WBC is the main author of this manuscript. He conceived the novel ideas, designed the algorithms and experiments, and performed the analysis. He wrote the entire manuscript. He accomplished all the revisions provided during entire peer review process until publication. He conducted the final proof reading as well. This manuscript is the outcomes of the research activities carried out only by the main author. KD and WC checked, reviewed the manuscript, and gave valuable suggestions on the structure of the paper. All authors have read approved the final manuscript.

\section{Competing interests}

The authors declare that they have no competing interests.

\section{Publisher's Note}

Springer Nature remains neutral with regard to jurisdictional claims in published maps and institutional affiliations.

\section{Author details}

${ }^{1}$ School of Physics and Electronic Engineering, Yibin University, Ybin 644000, China. ${ }^{2}$ Key Lab of Earth Exploration \& Information Techniques of Ministry of Education, Chengdu University of Technology, Chengdu 610059, China.

${ }^{3}$ Network and Multimedia Management Center, Yibin University, Ybin 644000 , China

Received: 21 October 2018 Accepted: 11 January 2019

Published online: 11 February 2019

References

1. H.T. Hsu, F.Y. Kuo, P.H. Lu, Design of WiFi/WiMAX dual-band E-shaped patch antennas through cavity model approach. Microw. Opt. Technol. Lett. 52(2), 471-474 (2010)

2. J. Xu, W. Wu, Compact microstrip dual-mode dual-band band-pass filters using stubs loaded coupled line. Prog. Electromagn. Res. C. 14, 137-150 (2013)

3. X.L. Peng, X.C.H. Wei, The resonance principle and designing method of multi-bands micro-strip antenna with branch shape. J. Antennas 2(4), 21-26 (2013)

4. W. Liu, M. Wang, W. Wu, Design on bi-directional circularly polarized microstrip antenna for mobile communication. J. Antennas 3(2), 9-14 (2014) 
5. D.Y. Wang, M. Wang, Y. Yuan, W. Wu, Design of broad band micro-strip antenna at S band. J. Antennas 4(3), 17-24 (2015)

6. Y. Yuan, M. Wang, D.Y. Wang, W. Wu, Design of broadband circularly polarized microstrip antenna at S band. J. Microwaves 33(4), 36-40 (2017)

7. S. Yang, J. Chen, K. Deng, Design of compact dual-band band-pass filter using dual-mode stepped-impedance stub resonators. Electron. Lett. 50(8), 611-613 (2014)

8. S.S. Gao, S. Sun, J.L. Li, Compact dual-mode dual-band band-pass filter with inside-outside-reversed dual-ring topology. Electron. Lett. 53(9), 624-626 (2017)

9. K. Zhou, C. Zhou, W. Wu, Substrate integrated waveguide dual-band filter with wide-stop band performance. Electron. Lett. 53(16), 1121-1123 (2017)

10. W.B. Cheng, Construction of WSN and application of localization for in-home healthcare of the elderly. Appl. Mech. Mater., vol 263-266 (2013), p. 943-947

11. W.B. Cheng, K. Deng, A novel indoor localization algorithm design for inhome healthcare of the elderly based on WSN. Open Cybern. Syst. J. 8(1), 1280-1285 (2014)

12. W.B. Cheng, W. Cheng, K. Deng, G. Guan, A tracking algorithm design for indoor mobile target based on EKF. J. Residuals Sci. Technol. 13(5), 133.1133.7 (2016)

13. W.B. Cheng, H.J. Wang, The key techniques of mine personnel localization system based on wireless sensor network. Microelectron. Comput. 29(4), 165-168 (2012)

14. G.L. Matthaei, L. Young, E.M.T. Jones. Microwave filters, impedance-Matching networks, and coupling structures. (Artechic House Publishers, 1980), p. 83723

15. J.S. Hong, M.J. Lancaster, Microstrip Filters for RF/Microwave Applications. (Wiley, 2001), p. 29-77

16. R.J. Cameron, C.M. Kudsia, R.R. Mansour.Microwave filters for communicationsystems: fundamentals, design and applications. (Wiley, 2007), p. 427-858

17. Y. Yin, M. Wang, R. Xu, W. Wu, Design of wideband harmonic rejection antenna. J. Antennas 6(1), 1-6 (2017)

18. N. Kumar, Y.K. Singh, Compact tri to dual pass-band switch-able band-pass filter using stub-loaded split-ring resonator with improved bandwidth Electron. Lett. 51(19), 1510-1512 (2015)

19. F. Wei, Y.J. Guo, X.W. Shi, Design of multi-band band-pass filters based on stub loaded stepped-impedance resonator with defected micro-strip structure. IET Microwaves, Antennas Propag. 10(2), 230-236 (2016)

20. J. Xu, Y. Zhu, Compact semi-lumped dual- and tri-wideband band-pass filters. IET Microwaves Antennas Propag. 11(1), 53-58 (2017)

21. P.C. Zhao, Z.Y. Zong, W. Wu, A fuss structure based on parallels resonators for multi-band applications. IEEE Trans. Antennas Propag. 65(10), 5257-5266 (2017)

22. X. Shang, Y. Wang, G.L. Nicholson, Design of multiple-pass-band filters using coupling matrix optimization. IET microwaves. Antennas Propag. 6(1), 24-30 (2012)

23. H. Zhang, W. Kang, W. Wu, Differential substrate integrated waveguide band-pass filter with improved common-mode suppression utilising complementary split-ring resonators. Electron. Lett. 53(7), 508-510 (2017)

24. S. Zhang, J.Y. Rao, J.J. Cheng, Novel compact single-band and dual-band band-pass filter based on one-third-mode substrate integrated waveguide. IEICE Electron. Express 14(19), 153-156 (2017)

25. M.D. Hickle, D. Peroulis, Tunable constant-bandwidth substrate-integrated band-stop filters. IEEE. Trans. Microwave Theory Tech. 66(1), 157-169 (2018)

26. B. Lee, B. Koh, S. Nam, Band-switchable substrate-integrated wave-guide resonator and filter. IEEE. Trans. Microwaves Theory Tech. 66(1), 147-156 (2018)

27. U. Naeem, M.B. Khan, M.F. Shafique, Design of compact dual-mode dualband SIW filter with independent tuning capability. Microw. Opt. Technol. Lett. 60(1), 178-182 (2018)

28. D. Bukuru, K. Song, F. Zhang, Compact quad-band band-pass filter using quad-mode stepped impedance resonator and multiple coupling circuits. IEEE. Trans. Microwaves Theory Tech. 65(3), 783-791 (2017)

29. S. Butterworth, On the theory of filter amplifiers. Exp. Wireless Eng. 7(6), 536-541 (1930)

30. D.D. Pang, Y. Xiong, M. He, L. Ji, J.L. Zhang, New quaint-band multimode filter with multiple transmission zeros. Electron. Components Mat. 36(10), 67-72 (2017)

31. Y.L. Zhang, T. Su, B. Wu, R.J. Zhou, Optimization synthesis technique for cross-coupled filter with frequency dependent couplings. J. Univ. Electron. Sci. Technol. China 47(4), 516-520 (2018)
32. J. Xu, W. Wu, G. Wei, Compact multi-band band-pass filters with mixed electric and magnetic coupling using multiple-mode resonator. IEEE Trans. Microwave Theory Tech. 63(12), 3909-3919 (2015)

33. F. Zhang, Y. Xiong, W. Zhang, D.D. Pang, X. Zhang, Tunable band pass filter based on cross-coupled structure. Electron. Components Mat. 37(5), 67-72 (2018)

34. C.H. Sun, T. Feng, Cross-coupled bandpass filter with microstrip lines. Electron. Components Mat. 35(12), 54-56 (2016)

35. R.B. Ma, J.G. Yan, X.W. Chen, L.Y. Yan, L.P. Han, Design of the novel miniaturized cross-coupled band-pass filter. J. Test Meas. Technol. 30(1), 6973 (2016)

36. R.J. Cameron, Fast generation of Chebyshev filter prototypes with asymmetrically-prescribed transmission zeros. ESA J. 6(1), 83-95 (1982)

37. R.J. Cameron, General coupling matrix synthesis methods for Chebyshev filtering function. IEEE. Trans. Microwave Theory Tech. 47(4), 433-443 (1999)

38. R.J. Cameron, Advanced coupling matrix synthesis techniques for microwave filters. IEEE. Trans. Microwave Theory Tech. 51(1), 1-10 (2003)

\section{Submit your manuscript to a SpringerOpen ${ }^{\circ}$ journal and benefit from:}

- Convenient online submission

- Rigorous peer review

- Open access: articles freely available online

- High visibility within the field

- Retaining the copyright to your article

Submit your next manuscript at $\boldsymbol{\nabla}$ springeropen.com 